\title{
Actualisation des tables de densité des ensilages de maïs
}

\author{
G Corrot \\ Institut de l'Elevage, 149 rue de Bercy, 75595 Paris Cedex 12, France
}

Les tables de prédiction de la densité ( $D$ en $\mathrm{kg}$ de $M S / m^{3}$ ) des ensilages de maïs utilisées depuis 1976 et basées sur une relation linéaire entre la densité d'une part et la teneur en matière sèche (MS) du maïs et la hauteur du silo d'autre part, ne sont plus adaptées aux conditions actuelles : faible précision et surestimation liée à l'extrapolation à des maïs plus riches en MS, à des silos plus hauts. II est donc nécessaire de proposer d'autres tables. Pour cela, l'étude a porté sur 394 silos couloirs (réalisés par 13 EDE en 3 campagnes) et 192 silos taupinières (EDE des Pays de Loire en 3 campagnes).

Les analyses et enregistrements ont concerné : la densité brute sur front d'attaque (densité en $\mathrm{kg} / \mathrm{m}^{3}$ calculée à partir de la moyenne de 12 carottages horizontaux de $35 \mathrm{~cm}$ de long sur 6 $\mathrm{cm}$ de diamètre), la densité brute de dessus de silo ( $\mathrm{CV}$ en $\mathrm{kg} / \mathrm{m}^{3}$ mesurée sur une carotte verticale de $50 \mathrm{~cm}$ de hauteur et de $6 \mathrm{~cm}$ de diamètre), les teneurs en matière sèche (MS en $\%$ ), NDF ( $\% M S$ ), en amidon ( $A$ en $\%$ de $M S)$, la hauteur du front de coupe ( $\mathrm{H}$ en mètre), les granulométries 10 et $20 \mathrm{~mm}$ (proportions du poids brut de l'ensilage qui sont respectivement retenues sur des grilles de 10 et $20 \mathrm{~mm}$ ), l'état végétatif (dessèchement...), les critères d'expression du tassement (mode de remplissage, durée, débit, chargement, coefficient synthétique). Les modélisations ont été établies par régression multiple, les critères explicatifs sont retenus au risque $5 \%$.

Le prisme est une méthode représentative de la densité du front de dessilage et peut être retenu comme témoin. La méthode de carottage horizontal est la méthode de terrain la plus aisée à mettre en oeuvre (temps nécessaire : 30 à 50 min pour une personne) et aussi la plus fiable. Elle est juste après application d'un coefficient de correction de 1,03 . Sa répétabilité est supérieure à celle du prisme : précisions respectives : $\pm 2,5 \%$ et $\pm 7,0 \%$.

C'est avec la densité exprimée en $\mathrm{kg}$ brut $/ \mathrm{m}^{3}$ que l'on observe les meilleures liaisons. Les critères explicatifs sont par ordre d'importance : CV, MS, A ou NDF, $H$. Les critères MS et $\mathrm{H}$ n'expriment à eux seuls que $45 \%$ de la variance brute (formule 1) confirmant la bibliographie (Bureau Commun inter Instituts, EDE, 1973). Parmi les critères cherchant à quantifier la résistance à la compression, la granulométrie et l'état de végétation apparaissent indépendants, par contre les teneurs en NDF ou en amidon expliquent une part de la variance brute presque égale à celle expliquée par la teneur MS (formules 2 et 3 ). L'ajout du critère $\mathrm{CV}$ (formule 6) permet d'expliquer les $3 / 4$ de la variabilité totale. La précision, au risque $5 \%$, reste encore médiocre ; pour une valeur de densité donnée, la prédiction peut varier dans l'intervalle de confiance (IC) de $\pm 34 \mathrm{~kg}$ de MS. Les critères d'expression du tassement n'ont jamais été significatifs. A caractéristiques comparables la densité du silo taupinière est inférieure à celle du silo couloir (formule 7).

Ce travail contribue à améliorer la connaissance des facteurs régissant la densité des ensilage de maïs (compressibilité). L'amidon et la carotte verticale supérieure améliorent sensiblement la fiabilité des prédictions. Les formules 3 et 5 ont été vulgarisées sous forme de tables ; l'une ou l'autre peut être utilisée selon que l'on dispose ou non de la teneur en amidon pour permettre un bilan des stocks avant ouverture. La précision de la prédiction individuelle reste toutefois médiocre. Une mesure directe sur le silo en exploitation doit permettre son ajustement.

Formules :

$$
\begin{aligned}
& 1-D \mathrm{~kg} \mathrm{MS} / \mathrm{m}^{3}=(934-11,9 \mathrm{MS}+57 \mathrm{H}) \mathrm{MS} / 100 \\
& 2-\mathrm{D}=(1429-15,0 \mathrm{MS}-8,25 \mathrm{NDF}+47 \mathrm{H}) \mathrm{MS} / 100 \\
& 3-\mathrm{D}=(929-17,5 \mathrm{MS}+7,9 \mathrm{~A}+44 \mathrm{H}) \mathrm{MS} / 100 \\
& 4-\mathrm{D}=(0,85 \mathrm{CV}+202) \mathrm{MS} / 100 \\
& 5-\mathrm{D}=(666+0,55 \mathrm{CV}-10,3 \mathrm{MS}+30 \mathrm{H}) \mathrm{MS} / 100 \\
& 6-\mathrm{D}=(606+0,45 \mathrm{CV}-12,3 \mathrm{MS}+5,5 \mathrm{~A}+35 \mathrm{H}) \mathrm{MS} / 100 \\
& 7-\mathrm{D}=(874-14,5 \mathrm{MS}+4,2 \mathrm{~A}+48 \mathrm{H}) \mathrm{MS} / 100
\end{aligned}
$$

$$
\begin{aligned}
& \mathrm{R}^{2}=0,45-\mathrm{IC}= \pm 47 \\
& \mathrm{R}^{2}=0,63-\mathrm{IC}= \pm 40 \\
& \mathrm{R}^{2}=0,66-\mathrm{IC}= \pm 37 \\
& \mathrm{R}^{2}=0,54-\mathrm{IC}= \pm 45 \\
& \mathrm{R}^{2}=0,69-\mathrm{IC}= \pm 37 \\
& \mathrm{R}^{2}=0,75-\mathrm{IC}= \pm 34 \\
& \mathrm{R}^{2}=0,54-\mathrm{IC}= \pm 44
\end{aligned}
$$

